\title{
Effects of diabetes on the rebleeding rate following endoscopic treatment in patients with liver cirrhosis
}

\author{
XI WANG ${ }^{1}, \mathrm{XUECAN} \mathrm{MEI}^{1}$ and DERUN KONG ${ }^{1,2}$ \\ ${ }^{1}$ Department of Gastroenterology, The First Affiliated Hospital of Anhui Medical University, Hefei, Anhui 230022; \\ ${ }^{2}$ Department of Gastroenterology, Fuyang Hospital of Anhui Medical University, Fuyang, Anhui 236000, P.R. China
}

Received August 2, 2019; Accepted December 9, 2019

DOI: $10.3892 / \mathrm{etm} .2020 .8876$

\begin{abstract}
In the present study, the effects of diabetes on rebleeding following endoscopic treatment were assessed in patients with liver cirrhosis. A retrospective analysis of patients who underwent endoscopic variceal ligation (EVL) or endoscopic injection sclerotherapy (EIS) at the First Affiliated Hospital of Anhui Medical University (Hefei, China) between June 2015 and March 2018 was performed. The patients were divided into the EVL and the EIS groups and each group was subdivided into diabetic and non-diabetic groups. The post-operative rebleeding rate was compared between the EVL and the EIS groups and between the diabetic and non-diabetic patients. The differences in the post-operative rebleeding rate of diabetic patients with hepatogenic and non-hepatogenic diabetes and in patients with different liver function grades were also determined. In the total patient cohort, the rebleeding rate in the EVL subgroup (11.3, 16.5 and 23.5\%) was not significantly different compared with that in the EIS subgroup $(9.8,17.4$ and $29.3 \%)$ at 1,3 and 6 months following surgery, respectively $(\mathrm{P}=0.724,0.868$ and 0.339$)$. In the total diabetic group, the rebleeding rate in the EVL subgroup (25.0, 36.1 and $44.4 \%$ ) was not significantly different compared with that in the EIS subgroup (20.6, 32.4 and 47.1\%) at 1, 3 and 6 months following surgery $(\mathrm{P}=0.660,0.741$ and 0.826 , respectively). In the EVL group, the rebleeding rate in the diabetic subgroup $(25.0,36.1$ and $44.4 \%)$ was higher than that in the non-diabetic subgroup (5.1, 7.6 and 13.9\%) at 1, 3, and 6 months following surgery and the differences were significant $(\mathrm{P}=0.005,<0.001$ and $<0.001$, respectively). In the EIS group, the rebleeding rate in the diabetic subgroup (20.6, 32.4 and $47.1 \%)$ was significantly higher than that in the non-diabetic subgroup (3.4, 8.6 and $19.0 \%)$ at 1,3 and 6 months following surgery $(\mathrm{P}=0.021$, 0.004 and 0.004 , respectively). Adjustment for age and liver
\end{abstract}

Correspondence to: Dr Derun Kong, Department of Gastroenterology, The First Affiliated Hospital of Anhui Medical University, 218 Jixi Road, Hefei, Anhui 230022, P.R. China

E-mail: kongderun168@163.com

Key words: liver cirrhosis, diabetes, esophageal varices, endoscopic treatment, ligation, sclerotherapy function grade in the EVL and EIS groups was performed using binary logistic regression and the parameter diabetes was indicated to be a risk factor for post-operative rebleeding $(\mathrm{P}<0.05)$. No significant difference was noted in the rate of rebleeding between patients with hepatogenic diabetes and non-hepatogenic diabetes at 1, 3 and 6 months following surgery $(\mathrm{P}=0.634,0.726$ and 0.446 , respectively). In the total diabetic group, the rebleeding rate in the Child-Pugh grade A subgroup (14.3, 17.9 and 25.0\%) was lower than that in the Child-Pugh grade B/C subgroup (28.6, 45.2 and 59.5\%) at 1, 3 and 6 months following surgery, respectively. No significant difference was noted between the two groups at 1 month following surgery $(\mathrm{P}=0.163)$. However, the differences were significant at 3 and 6 months following surgery $(\mathrm{P}=0.018$ and 0.005 , respectively). The results suggested that diabetes is a risk factor for post-operative rebleeding in patients with cirrhosis. Diabetic patients with poor liver function were more likely to bleed following surgery and the post-operative bleeding rate was not significantly different between patients with hepatogenic and non-hepatogenic diabetes. The study was registered in the Chinese Clinical Trial Registry (no. ChiCTR1800017772).

\section{Introduction}

Esophageal variceal bleeding (EVB) is a serious complication of liver cirrhosis that affects the life of patients and has a high mortality rate (1). Endoscopic variceal ligation (EVL) and endoscopic injection sclerotherapy (EIS) are effective methods for preventing and treating $\operatorname{EVB}(2,3)$. These two methods have become the first-line treatment for EVB (4). However, the incidence of post-operative rebleeding is a major concern. Previous studies have indicated that risk factors for rebleeding following endoscopic treatment include the following: Varicose vein severity, Child-Pugh grade C, ascites volume (medium-mass), portal vein thrombosis and previous history of diabetes (5). It is important to prevent rebleeding following endoscopic treatment and improve the prognosis of patients. The present study demonstrated that in addition to liver function-associated factors, diabetes is associated with an increased prevalence of post-operative bleeding. The control of repeated bleeding in these patients remains a clinical challenge. Therefore, further studies are required to explore the factors that are associated with post-operative rebleeding in order to evaluate the effects 
of diabetes on this complication that is encountered following endoscopic treatment. Such investigations may lead to developments that may improve the prognosis of patients with cirrhosis and diabetes. In the present study, a retrospective analysis of risk factors for post-operative rebleeding in patients with cirrhosis, EVB and diabetes was performed.

\section{Materials and methods}

Patients. The data were obtained from patients who underwent EVL or EIS for the prevention or treatment of EVB at the Department of Gastroenterology, the First Affiliated Hospital of Anhui Medical University (Hefei, China). The patients were enrolled between June 2015 and March 2018. The treatment provided to the patients did not include EVL and EIS simultaneously. The inclusion criteria were as follows: i) Patients aged 18-75 years; ii) patients diagnosed with cirrhosis and esophageal varices without gastric varices; iii) history of variceal bleeding and endoscopic sclerotherapy or ligation. The exclusion criteria were as follows: i) Patients aged $<18$ or $>75$ years; ii) gastrointestinal bleeding at other sites or due to other reasons; iii) blood system diseases; iv) other serious organ complications and/or malignant tumors; v) history of interventional or surgical treatment. A total of 207 patients were enrolled, including 140 males and 67 females. The average age was 53.31 \pm 9.76 years (range, 25-74 years). A total of 137 patients were diabetic and 70 patients were non-diabetic. According to the diagnostic criteria for hepatogenic diabetes mellitus (6), the patients had no family history of diabetes. Diabetes was diagnosed following liver cirrhosis or at the same time as liver cirrhosis. A total of 2 patients with an unclear medical history were excluded and 33 of the remaining 68 patients exhibited hepatogenic diabetes. The causes of cirrhosis were the following: Post-hepatitis B $(\mathrm{n}=111)$, post-hepatitis $\mathrm{C}(\mathrm{n}=6)$, alcoholic cirrhosis $(\mathrm{n}=23)$, cholestatic cirrhosis $(\mathrm{n}=10)$, cirrhosis following autoimmune hepatitis $(n=8)$, schistosomiasis cirrhosis $(n=6)$, cryptogenic cirrhosis $(n=20)$, Budd-Chiari syndrome $(n=2)$, non-alcoholic steatohepatitis-cirrhosis $(n=1)$ and mixed cirrhosis $(n=20)$.

The patients were divided into the EVL and the EIS groups and each group was subdivided into diabetic and non-diabetic subgroups. The EVL group comprised 115 patients, including 36 in the diabetic group and 79 in the non-diabetic group. A total of 92 patients were present in the EIS group, including 34 in the diabetic group and 58 in the non-diabetic group. All patients provided written informed consent prior to surgery. The present study was approved by the Ethics Committee of the First Affiliated Hospital of Anhui Medical University (Hefei, China) and was registered as a clinical trial.

Materials. An Olympus GIF-XQ260 electronic gastroscope (Olympus) and an adjustable disposable needle (23G; Cook Co.) were used; the sclerosant (lauromacrogol) was obtained from Shanxi Tianyu Co., and the multiband ligation device (6-ring ligation device; Cook Co.) was used.

Treatment. The patients were routinely administered pre-operative treatment to reduce portal pressure and correct anemia. A comprehensive assessment of the patients prior to

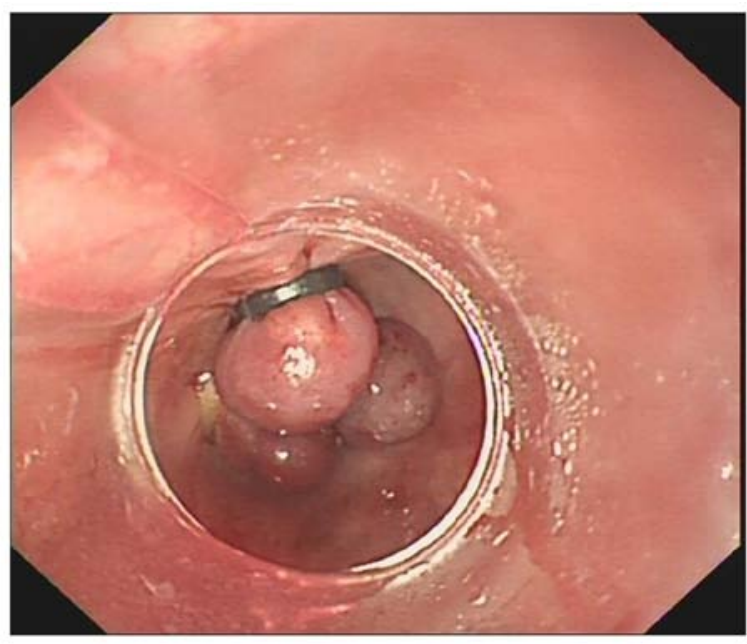

Figure 1. Representative intra-operative image of endoscopic ligation.

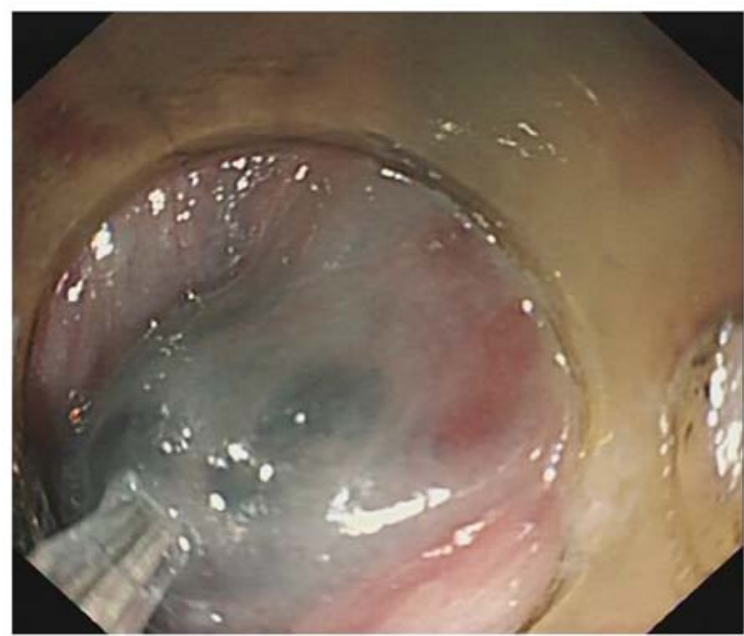

Figure 2. Representative intra-operative image of endoscopic injection sclerotherapy.

surgery was performed to ensure that their vital signs were stable. The patients underwent routine gastroscopy prior to surgery to determine the position, shape, number and size of the varicose veins. The medical practitioner decided on the number of rings used for EVL or the amount of the sclerosant required, and the gastroscope was subsequently removed. The endoscopic treatment plan for each patient was discussed by three doctors. Following confirmation, the senior endoscopic expert completed the procedure.

In the EVL group, ligation was performed from above or below the dentate line, indicating the region where the esophageal varicose vein was formed. The ligation point was selected and the endoscope end was placed close to the varicose vein. Negative pressure was used until the field of view was completely red. The second ring was placed immediately adjacent to the previous ring and ligation was performed from the top using the same method (Fig. 1).

In the EIS group, a range of 2-7 points was used per injection according to the severity of the varicose veins. The total amount did not exceed $40 \mathrm{ml}$, with $0.5-10 \mathrm{ml}$ per point (lauromacrogol $10 \mathrm{ml}+$ meilan $0.1 \mathrm{ml}$, until the filling of 
Table I. Basic characteristics of the two groups of patients with cirrhosis.

\begin{tabular}{|c|c|c|c|c|c|c|}
\hline \multirow[b]{2}{*}{ Item } & \multicolumn{3}{|c|}{ EVL group } & \multicolumn{3}{|c|}{ EIS group } \\
\hline & $\begin{array}{c}\text { Diabetic } \\
\text { group }(n=36)\end{array}$ & $\begin{array}{l}\text { Non-diabetic } \\
\text { group }(n=79)\end{array}$ & P-value & $\begin{array}{c}\text { Diabetic } \\
\text { group }(n=34)\end{array}$ & $\begin{array}{l}\text { Non-diabetic } \\
\text { group }(\mathrm{n}=58)\end{array}$ & P-value \\
\hline Age (years) & $55.78 \pm 8.98$ & $53.80 \pm 10.09$ & 0.315 & $57.03 \pm 9.28$ & $48.93 \pm 8.59$ & $<0.001$ \\
\hline Sex (male/female) & $27 / 9$ & $45 / 34$ & 0.064 & $26 / 8$ & $42 / 16$ & 0.669 \\
\hline Hemoglobin (g/l) & $85.50 \pm 24.80$ & $83.21 \pm 20.61$ & 0.606 & $75.38 \pm 21.86$ & $78.91 \pm 23.52$ & 0.478 \\
\hline Platelet $\left(10^{9} / 1\right)$ & $87.42 \pm 69.94$ & $104.94 \pm 93.59$ & 0.318 & $93.74 \pm 68.98$ & $108.81 \pm 70.77$ & 0.320 \\
\hline White blood cell $\left(10^{9} / 1\right)$ & $3.70 \pm 2.06$ & $3.68 \pm 2.42$ & 0.966 & $3.95 \pm 2.15$ & $4.11 \pm 2.90$ & 0.769 \\
\hline Albumin $(\mathrm{g} / \mathrm{l})$ & $35.07 \pm 5.45$ & $34.10 \pm 6.02$ & 0.969 & $34.48 \pm 3.89$ & $35.49 \pm 6.74$ & 0.364 \\
\hline Child-Pugh grade (A/B/C) & $15 / 17 / 4$ & $45 / 32 / 2$ & 0.088 & $13 / 16 / 5$ & $38 / 18 / 2$ & 0.019 \\
\hline MELD scores & $7.22 \pm 3.98$ & $8.29 \pm 2.35$ & 0.141 & $8.71 \pm 2.89$ & $7.88 \pm 2.14$ & 0.120 \\
\hline Esophageal varices (medium/heavy) & $3 / 33$ & $3 / 76$ & 0.375 & $4 / 30$ & $10 / 48$ & 0.480 \\
\hline Red color & $33 / 36$ & $72 / 79$ & 0.926 & $33 / 34$ & $57 / 58$ & 0.699 \\
\hline Diameter of EVs (cm) & $0.967 \pm 0.13$ & $0.929 \pm 0.15$ & 0.192 & $0.935 \pm 0.14$ & $0.955 \pm 0.13$ & 0.489 \\
\hline Portal vein thrombosis & $10 / 26$ & $17 / 62$ & 0.463 & $9 / 25$ & $18 / 39$ & 0.606 \\
\hline
\end{tabular}

Values are expressed as $\mathrm{n}$ or the mean \pm standard deviation. EIS, endoscopic injection sclerotherapy; EVL, endoscopic variceal ligation; EV, esophageal varix; MELD, model for end-stage liver disease.

all varicose veins was satisfactory). The patients were fasted for $24 \mathrm{~h}$ following surgery and rehydrated. The secretion of gastric acid was inhibited, the portal pressure was reduced and prophylactic anti-infective treatment was administered. At 24-48 $\mathrm{h}$ following surgery, the liquid diet was gradually changed to a semi-liquid diet and subsequently to a soft food diet. The patients' daily diet was mainly composed of soft food. Strenuous exercise was avoided. A representative image of EIS is presented in Fig. 2.

Follow-up and analysis. At 1, 3 and 6 months following surgery, the patients were followed up by gastroscopy to detect bleeding and determine whether the varicose veins had disappeared. If the varicose veins had not completely disappeared, consolidation treatment was performed. The post-operative rebleeding rates were compared between the EVL and EIS subgroups of the total patient group and the total diabetic group. The rebleeding rate in the diabetic and non-diabetic patients of the EVL and EIS groups was analyzed at 1, 3 and 6 months following surgery. The influence of the parameters of age and liver function grade in the diabetic and non-diabetic patients in the EIS group was controlled by binary logistic regression. The post-operative rebleeding rate was compared between the patients with hepatogenic and non-hepatogenic diabetes from the total diabetic group. The total diabetic group was classified into the Child-Pugh A and Child-Pugh $\mathrm{B}+\mathrm{C}$ groups according to the liver function grade. The rebleeding rate in the patients with diabetes in the Child-Pugh $\mathrm{A}$ and Child-Pugh B/C groups was analyzed at 1,3 and 6 months following surgery to observe whether liver function grade had an influence on rebleeding in patients with cirrhosis and diabetes.

Statistical analysis. Statistical analysis was performed using the SPSS 19.0 software (IBM Corp.). The measurement data that followed a normal distribution were expressed as the mean \pm standard deviation and comparisons between the two groups were performed using the independent-samples t-test. The enumeration data were expressed by the number of cases and the rebleeding rate. The comparisons between the two groups were performed using the $\chi^{2}$ test. The influence of the confounding factors was controlled by using binary logistic regression. Kaplan-Meier curve analysis and the log-rank test were used to compare the rebleeding rates at different durations between the two groups. The level of significance was set as $\alpha=0.05$. $P<0.05$ was considered to indicate a statistically significant difference.

\section{Results}

Baseline data analysis. The baseline data of the patients in the EVL and the EIS groups were collected and the diabetic and non-diabetic subgroups of the EVL group did not exhibit any significant differences. In the EIS group, the diabetic patients were older than the non-diabetic patients and the number of patients with liver function grades B and C was higher. The differences noted were significant. No significant differences were evident in the baseline data regarding gender, hemoglobin, varicose veins and the presence or absence of portal vein thrombosis (Table I). In the total diabetic group, no significant differences were noted in the baseline data between patients with hepatogenic diabetes and non-hepatogenic diabetes.

Post-operative rebleeding rate in the EVL and EIS groups in the total patient group and the total diabetic group. Comparisons of the post-operative rebleeding rate in the EVL and EIS groups in the total patient group and the total diabetic group are provided in Table II. In the total patient group, the 
Table II. Post-operative rebleeding rate of the EVL and EIS subgroups of the total patient and the total diabetic group.

\begin{tabular}{|c|c|c|c|c|}
\hline \multirow[b]{2}{*}{ Time-point/rebleeding } & \multicolumn{2}{|c|}{ Total patients } & \multicolumn{2}{|c|}{ Total diabetic patients } \\
\hline & EVL group & EIS group & EVL group & EIS group \\
\hline \multicolumn{5}{|l|}{1 month } \\
\hline Yes & $13(11.3)$ & $9(9.8)$ & $9(25)$ & $7(20.6)$ \\
\hline No & $102(88.7)$ & $83(90.2)$ & $27(75)$ & $27(79.4)$ \\
\hline P-value & 0.724 & & 0.660 & \\
\hline \multicolumn{5}{|l|}{3 months } \\
\hline Yes & $19(16.5)$ & $16(17.4)$ & $13(36.1)$ & $11(32.4)$ \\
\hline No & $96(83.5)$ & $76(82.6)$ & $23(63.9)$ & $23(67.6)$ \\
\hline P-value & 0.868 & & 0.741 & \\
\hline \multicolumn{5}{|l|}{6 months } \\
\hline Yes & $27(23.5)$ & $27(29.3)$ & $16(44.4)$ & $16(47.1)$ \\
\hline No & $88(76.5)$ & $65(70.7)$ & $20(55.6)$ & $18(52.9)$ \\
\hline P-value & 0.339 & & 0.826 & \\
\hline
\end{tabular}

Values are expressed as n (\%). EIS, endoscopic injection sclerotherapy; EVL, endoscopic variceal ligation.

Table III. Comparison of rebleeding rates between diabetic and non-diabetic patients.

\begin{tabular}{|c|c|c|c|c|}
\hline \multirow[b]{2}{*}{ Time-point/rebleeding } & \multicolumn{2}{|c|}{ EVL group } & \multicolumn{2}{|c|}{ EIS group } \\
\hline & Diabetic group & Non-diabetic group & Diabetic group & Non-diabetic group \\
\hline \multicolumn{5}{|l|}{1 month } \\
\hline Yes & $9(25.0)$ & $4(5.1)$ & 7 (20.6) & $2(3.4)$ \\
\hline No & $27(75.0)$ & $75(94.9)$ & $27(79.4)$ & $56(96.6)$ \\
\hline P-value & 0.005 & & 0.021 & \\
\hline \multicolumn{5}{|l|}{3 months } \\
\hline Yes & $13(36.1)$ & $6(7.6)$ & $11(32.4)$ & $5(8.6)$ \\
\hline No & $23(63.9)$ & $73(92.4)$ & $23(67.6)$ & $53(91.4)$ \\
\hline P-value & $<0.001$ & & 0.004 & \\
\hline \multicolumn{5}{|l|}{6 months } \\
\hline Yes & $16(44.4)$ & $11(13.9)$ & $16(47.1)$ & $11(19.0)$ \\
\hline No & $20(55.6)$ & $68(86.1)$ & $18(52.9)$ & $47(81.0)$ \\
\hline P-value & $<0.001$ & & 0.004 & \\
\hline
\end{tabular}

Values are expressed as n (\%). EIS, endoscopic injection sclerotherapy; EVL, endoscopic variceal ligation.

rebleeding rate in the EVL subgroup $(11.3,16.5$ and $23.5 \%)$ was not significantly different compared with that in the EIS subgroup $(9.8,17.4$ and $29.3 \%)$ at 1,3 and 6 months following surgery $(\mathrm{P}=0.724,0.868$ and 0.339 , respectively). In the total diabetic group, the rebleeding rate in the EVL group (25.0,36.1 and $44.4 \%$ ) was not significantly different compared with that in the EIS group (20.6, 32.4 and 47.1\%) at 1, 3 and 6 months following surgery $(\mathrm{P}=0.660,0.741$ and 0.826 , respectively).

Rebleeding rates in diabetic and non-diabetic patients and in hepatogenic and non-hepatogenic diabetic patients. First, the rebleeding rates were compared between diabetic and non-diabetic patients (Table III). In the EVL group, the rebleeding rate in the diabetic subgroup was $25.0 \%(9 / 36)$, $36.1 \%(13 / 36)$ and $44.4 \%$ (16/36) at 1, 3 and 6 months following surgery, respectively. The rebleeding rates in the non-diabetic group were $5.1 \%$ (4/79), $7.6 \%(6 / 79)$ and $13.9 \%(11 / 79)$ at 1,3 and 6 months following surgery, respectively. The difference between the two groups was significant $(\mathrm{P}=0.005,<0.001$ and 0.001 , respectively; Table III).

In the EIS group, the rebleeding rate in the diabetic subgroup was $20.6 \%$ (7/34), $32.4 \%$ (11/34) and $47.1 \%(16 / 34)$ at 1,3 and 6 months following surgery, respectively. The rebleeding rate in the non-diabetic group was $3.4 \%(2 / 58)$, 


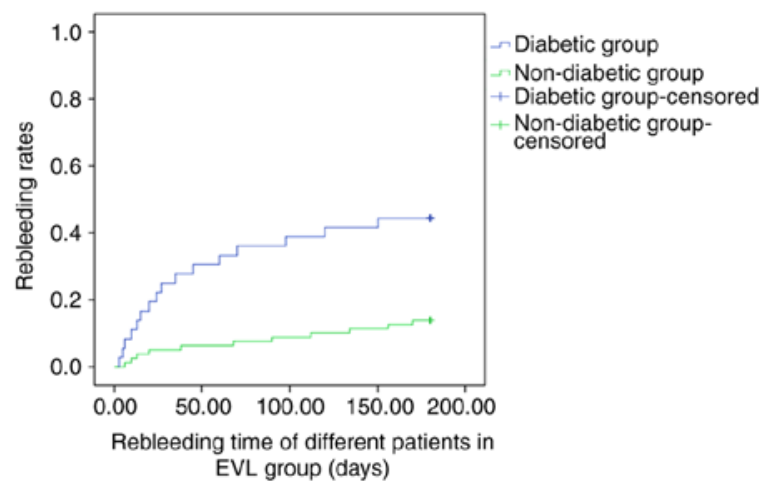

Figure 3. Kaplan-Meier curves of time-dependent rebleeding rates in diabetic and non-diabetic patients in the EVL group. EVL, endoscopic variceal ligation.

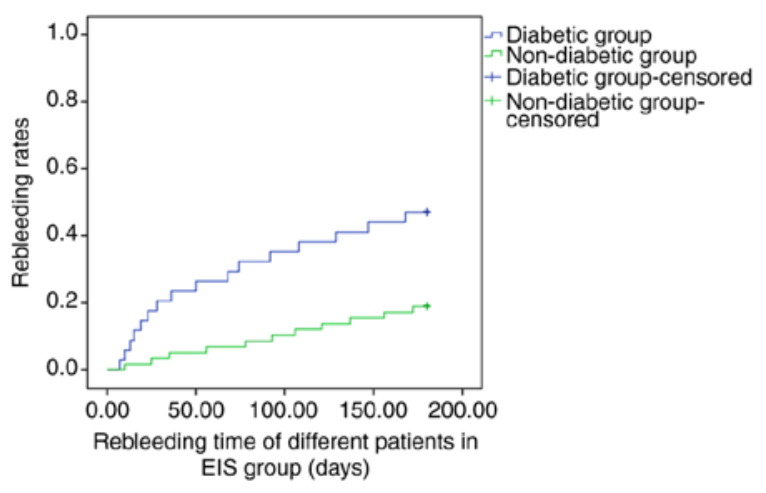

Figure 4. Kaplan-Meier curves of time-dependent rebleeding rates in diabetic and non-diabetic patients in the EIS group. EIS, endoscopic injection sclerotherapy.

$8.6 \%(5 / 58)$ and $19.0 \%(11 / 47)$ at 1,3 and 6 months following surgery, respectively. The difference between the two groups was significant $(\mathrm{P}=0.021,0.004$ and 0.004 , respectively; Table III).

Binary logistic regression was used to control for age and liver function grade (Table IV). The analysis indicated that diabetes was a risk factor for post-operative rebleeding. In the EVL group, the odds ratio (OR) with $95 \%$ CI was 5.518 (1.535-19.838), 5.861 (1.951-17.612) and 4.294 (1.668-11.054) at 1,3 and 6 months following surgery $(\mathrm{P}=0.004,<0.001$, 0.001 , respectively). During the first month of treatment, the EIS group exhibited an OR (95\% CI) of 5.900 (0.997-34.929, $\mathrm{P}=0.05)$ and at 3 and 6 months, the OR $(95 \% \mathrm{CI})$ was 3.809 (1.053-13.770) and 3.878 (1.333-11.284), respectively $(\mathrm{P}=0.041$, 0.013 ; Table IV).

A comparison of rebleeding rates between patients with hepatogenic and non-hepatogenic diabetes is provided in Table V. The rebleeding rate in patients with hepatogenic diabetes was $18.2 \%(6 / 33), 30.3 \%(10 / 33)$ and $39.4 \%(13 / 33)$ at 1,3 and 6 months following surgery, respectively. The rebleeding rate in patients with non-hepatogenic diabetes was $22.9 \%(8 / 35), 34.3 \%(12 / 35)$ and $48.6 \%(17 / 35)$ at 1,3 and 6 months following surgery, respectively. No significant difference was noted between the two groups at the different time-points $(\mathrm{P}=0.634,0.726$ and 0.446 , respectively; Table V).
Kaplan-Meier curve analysis and the log-rank test was used to compare the rebleeding rates after different time periods between diabetic and non-diabetic groups. Diabetes was associated with a higher bleeding rate and the difference between diabetic and non-diabetic groups was significant in the EVL and the EIS groups $(\mathrm{P}<0.001$ and 0.002; Figs. 3 and 4, respectively).

Post-operative rebleeding rates in diabetic patients with different liver function grades. The total patient group comprised 70 diabetic patients, including 28 (40\%) with liver function Child-Pugh grade A and $42(60 \%)$ with Child-Pugh grade of B/C. A total of 137 non-diabetic patients, $83(60.6 \%)$ with liver function Child-Pugh grade A and 54 (39.4\%) with Child-Pugh grade $\mathrm{B} / \mathrm{C}$ were enrolled. The difference between the two groups was significant $(\mathrm{P}=0.005)$. In the total diabetic group, the rate of rebleeding in the Child-Pugh grade A subgroup was $14.3 \%(4 / 28), 17.9 \%(5 / 28)$ and $25.0 \%(7 / 28)$ at 1,3 and 6 months following surgery, respectively. The rebleeding rate in the Child-Pugh grade $\mathrm{B} / \mathrm{C}$ group was $28.6 \%$ $(12 / 42), 45.2 \%(19 / 42)$ and $59.5 \%(25 / 42)$ at 1,3 and 6 months following surgery, respectively. The differences between the two groups were not significant at 1 month following surgery $(\mathrm{P}=0.163)$, whereas they were significant at 3 and 6 months following surgery, respectively $(\mathrm{P}=0.018$ and 0.005 , respectively; Table VI).

A patient with cirrhosis and diabetes suffered from major bleeding on the 10th day after EVL. In the present study, one patient with cirrhosis and diabetes suffered from major bleeding due to shedding of the apron that occurred 10 days following EVL. The patient was re-admitted to the First Affiliated Hospital of Anhui Medical University. Following compression of the Sengstaken-Blakemore tube, the patient's bleeding stopped. Gastroscopy revealed multiple large, unhealed ulcers in the esophagus (Fig. 5). To prevent rebleeding, small blood vessels around the ulcer were treated with sclerotherapy (Fig. 6).

\section{Discussion}

Endoscopic treatment is an effective method for EVB. The emergency hemostasis rate may reach more than $90 \%$ (2), which may effectively prevent rebleeding of patients. This method has become a mainstay of treatment for esophageal varices. However, the post-operative rebleeding rate is estimated at $\sim 20 \%$, which frequently affects patient survival (4). Previous studies have suggested that liver function Child-Pugh grade $\mathrm{B}$ or $\mathrm{C}$, ascites, varicose vein severity, coagulation function and diabetes are risk factors for rebleeding following endoscopic treatment (5). These factors are associated with the prognosis and mortality rate of patients. Hickman and Macdonald (7) reported that patients with liver cirrhosis and diabetes account for $\sim 30 \%$ of patients with liver cirrhosis. It was also indicated that diabetes may increase the incidence of complications and mortality among patients with cirrhosis (8). Qi et al (9) and Bai et al (10) reported that diabetes was a significant factor for predicting the short-term prognosis of patients with acute upper gastrointestinal bleeding and cirrhosis, and it was an independent predictor of in-hospital 


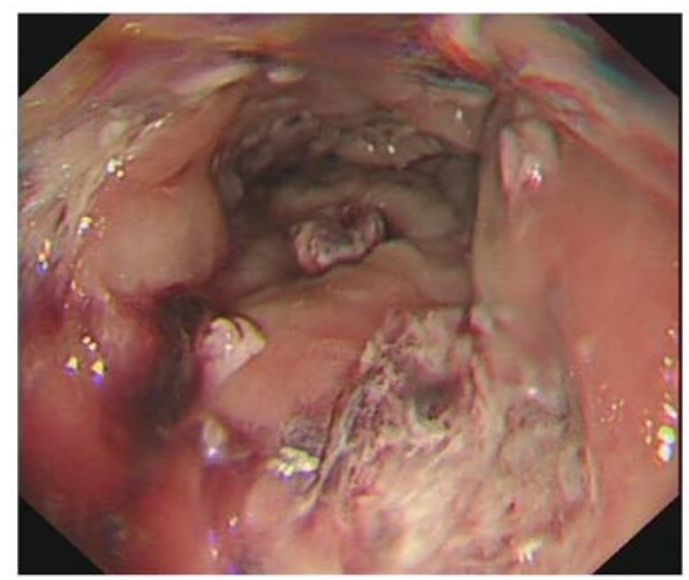

Figure 5. Gastroscopy indicating multiple large, unhealed ulcers in the esophagus.

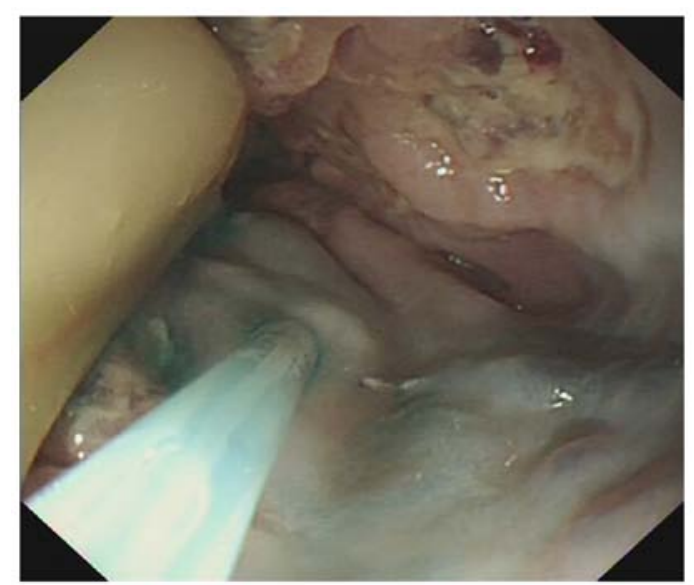

Figure 6. Small blood vessels around the ulcer were treated with sclerotherapy to prevent rebleeding.

death. The present retrospective study aimed to further clarify the effects of diabetes on rebleeding in patients with cirrhosis following endoscopic therapy.

The present results indicated that the post-operative rebleeding rate was significantly higher in the diabetic group compared with that in the non-diabetic group regardless of the type of treatment (EVL or EIS). These results indicated that patients with cirrhosis and diabetes exhibited a higher rate of post-operative rebleeding. However, in the EIS group, age and liver function grade at baseline were different in patients with diabetes vs. those of non-diabetic patients. The age of diabetic patients was higher than that of non-diabetic patients and the proportion of the liver function grades $\mathrm{B}$ and $\mathrm{C}$ was higher. To exclude the effects of these parameters on post-operative bleeding, age and liver function grade in the EVL and the EIS groups were controlled using binary logistic regression. The results indicated that in the EVL group, diabetes was a risk factor for rebleeding at 1,3 and 6 months following surgery. In the EIS group, diabetes was not a significant risk factor for rebleeding at 1 month following surgery (OR: 5.900, 95\% CI: 0.997-34.929; $\mathrm{P}=0.05$ ). This outcome was possibly associated with the small sample size $(\mathrm{n}=92)$. However, diabetes was a risk factor 
Table V. Comparison of rebleeding rates between patients with hepatogenic and non-hepatogenic diabetes.

\begin{tabular}{lcccccccc}
\hline & \multicolumn{2}{c}{1 month } & & \multicolumn{2}{c}{3 months } & & \multicolumn{2}{c}{6 months } \\
\cline { 2 - 3 } Pathology & Rebleeding & No rebleeding & & Rebleeding & No rebleeding & & Rebleeding & No rebleeding \\
\hline Hepatogenic diabetes & $6(18.2)$ & $27(81.8)$ & & $10(30.3)$ & $23(69.7)$ & & $13(39.4)$ & $20(60.6)$ \\
Non-hepatogenic diabetes & $8(22.9)$ & $27(77.1)$ & & $12(34.3)$ & $23(65.7)$ & & $17(48.6)$ & $19(51.4)$ \\
P-value & 0.634 & & & 0.726 & & & 0.446 & \\
\hline
\end{tabular}

Values are expressed as $\mathrm{n}(\%)$.

Table VI. Comparison of post-operative rebleeding rates in diabetic patients with different liver function grades.

\begin{tabular}{|c|c|c|c|c|c|c|}
\hline \multirow[b]{2}{*}{ Child-Pugh grade } & \multicolumn{2}{|c|}{1 month } & \multicolumn{2}{|c|}{3 months } & \multicolumn{2}{|c|}{6 months } \\
\hline & Rebleeding & No rebleeding & Rebleeding & No rebleeding & Rebleeding & No rebleeding \\
\hline A & $4(14.3)$ & $24(85.7)$ & $5(17.9)$ & $23(82.1)$ & $7(25.0)$ & $21(75.0)$ \\
\hline $\mathrm{B} / \mathrm{C}$ & $12(28.6)$ & $30(71.4)$ & $19(45.2)$ & $23(54.8)$ & $25(59.5)$ & $17(40.5)$ \\
\hline P-value & 0.163 & & 0.018 & & 0.005 & \\
\hline
\end{tabular}

Values are expressed as $\mathrm{n}(\%)$.

for rebleeding at 3 and 6 months following surgery. The Kaplan-Meier curve analysis and the log-rank test further indicated that the differences between the diabetic and the non-diabetic subgroups were significant in the EVL and the EIS groups. The post-operative rebleeding rates were not significantly different between the patients with hepatogenic and non-hepatogenic diabetes. Patients with diabetes exhibited a lower wound healing ability than healthy patients due to continuous hyperglycemia $(11,12)$. Therefore, the wound healing time was delayed in patients with cirrhosis and diabetes due to inadequate healing, irrespective of the endoscopic treatment used, resulting in increased risk of post-operative rebleeding.

The liver is an important organ of glucose metabolism, which may effectively maintain the stability of blood sugar in the human body. Liver cirrhosis damages normal liver cells, leading to disorders of glucose metabolism, including abnormal glucose tolerance or hepatogenic diabetes. Long-term hyperglycemia may further aggravate liver function damage and liver fibrosis $(13,14)$. In the present study, the proportion of patients in the diabetic group with a Child-Pugh grade B/C (60\%) was significantly higher than that of patients with Child-Pugh grade A $(40 \%)$ Patients with cirrhosis and diabetes exhibited reduced liver function compared with that of patients without diabetes. Hunter and Hamdy (15) indicated that patients with poor liver function and cirrhosis were more likely to develop rebleeding following surgery. The present study demonstrated that in the total diabetic patients, the rate of rebleeding in patients with Child-Pugh grade B/C (28.6\%) was higher than that in those with Child-Pugh grade A (14.3\%), although the differences were not significant. The rate of rebleeding in patients with Child-Pugh grade B/C at 3 and 6 months following surgery (45.2 and 59.5\%, respectively) was significantly higher than that in patients with Child-Pugh grade A (17.9 and $25.0 \%$, respectively). Furthermore, patients with poor liver function frequently had high-risk factors for a series of hemorrhages, including hypoalbuminemia, decreased blood coagulation and ascites. Therefore, it may be hypothesized that improvement of the liver function may in turn improve the overall condition of patients and reduce the risk of rebleeding.

In conclusion, the present study demonstrated that diabetes was a risk factor for post-operative rebleeding in patients with cirrhosis. Patients with cirrhosis and diabetes exhibited poor liver function and a high risk of post-operative rebleeding. Therefore, the treatment of such patients requires considerable attention in clinical practice. Blood glucose monitoring and improvement of liver function of patients prior to surgery are strict indications for the reduction of the risk of post-operative rebleeding by ligation treatment. The present study had certain limitations, including the small sample size, short follow-up duration and lack of investigation of the mechanism by which cirrhosis interacted with diabetes. Therefore, further studies are required to address these limitations.

\section{Acknowledgements}

Not applicable.

\section{Funding}

The present study was supported by the research fund project of the Anhui Provincial Institute of Translational Medicine 
(grant no. 2017zhyx 18) and the Anhui Science and Technology Department: 2018 Key Research and Development Plan Projects (grant no. 1804h08020260). The funders had no role in the study design, data collection and analysis, decision to publish, or preparation of the manuscript.

\section{Availability of data and materials}

The datasets used and/or analyzed during the present study are available from the corresponding author on reasonable request.

\section{Authors' contributions}

DK provided technical support and designed the study. XW performed the experiments, collected and analysed the data and wrote the manuscript. XM performed the experiments and collected and analysed the data. All authors read and approved the final manuscript.

\section{Ethics approval and consent to participate}

The study protocol was approved by the Ethics Committee of Anhui Medical University (Hefei, China) and all patients provided written informed consent prior to inclusion. The trial was registered in Chinese Clinical Trial Registry (Title: Effects of diabetes on the rebleeding rate following endoscopic treatment in patients with liver cirrhosis; no. ChiCTR1800017772).

\section{Patient consent for publication}

Not applicable.

\section{Competing interests}

The authors declare that they have no competing interests.

\section{References}

1. Kumar S, Asrani SK and Kamath PS: Epidemiology, diagnosis and early patient management of esophagogastric hemorrhage. Gastroenterol Clin North Am 43: 765-782, 2014.
2. Ali SM, Wu S, Xu H, Liu H, Hao J and Qin C: A prospective study of endoscopic injection sclerotherapy and endoscopic variceal ligation in the treatment of esophageal varices. J Laparoendosc Adv Surg Tech A 27: 333-341, 2017.

3. Zargar SA, Javid G, Khan BA, Shah OJ, Yattoo GN, Shah AH, Gulzar GM, Singh J, Shah NA and Shafi HM: Endoscopic ligation vs. sclerotherapy in adults with extrahepatic portal venous obstruction: A prospective randomized study. Gastrointest Endosc 61: 58-66, 2005.

4. Garcia-Tsao G, Abraldes JG, Berzigotti A and Bosch J: Portal hypertensive bleeding in cirrhosis: Risk stratification, diagnosis, and management: 2016 practice guidance by the American Association for the study of liver diseases. Hepatology 65: 310-335, 2017.

5. Xu L, Ji F, Xu QW and Zhang MQ: Risk factors for predicting early variceal rebleeding after endoscopic variceal ligation. World J Gastroenterol 17: 3347-3352, 2011.

6. Nishida T, Tsuji S, Tsujii M, Arimitsu S, Haruna Y, Imano E, Suzuki M, Kanda T, Kawano S, Hiramatsu N, et al: Oral glucose tolerance test predicts prognosis of patients with liver cirrhosis. Am J Gastroenterol 101: 70-75, 2006.

7. Hickman IJ and Macdonald GA: Impact of diabetes on the severity of liver disease. Am J Med 120: 829-834, 2007.

8. Quintana JO, García-Compean D, González JA, Pérez JZ, González FJ, Espinosa LE, Hernández PL, Cabello ER, Villarreal ER, Rendón RF and Garza HM: The impact of diabetes mellitus in mortality of patients with compensated liver cirrhosis-a prospective study. Ann Hepatol 10: 56-62, 2011.

9. Qi X, Peng Y, Li H, Dai J and Guo X: Diabetes is associated with an increased risk of in-hospital mortality in liver cirrhosis with acute upper gastrointestinal bleeding. Eur J Gastroenterol Hepatol 27: 476-477, 2015.

10. Bai Z, Li B, Lin S, Liu B, Li Y, Zhu Q, Wu Y, Yang Y, Tang S, Meng F, et al: Development and validation of CAGIB score for evaluating the prognosis of cirrhosis with acute gastrointestinal bleeding: A retrospective multicenter study. Adv Ther 36: 3211-3220, 2019.

11. Khangholi S, Majid FA, Berwary NJ, Ahmad F and Aziz RB: The mechanisms of inhibition of advanced glycation end products formation through polyphenols in hyperglycemic condition. Planta Med 82: 32-45, 2016.

12. Tian M, Qing C, Niu Y, Dong J, Cao X, Song F, Ji X and Lu S: The relationship between inflammation and impaired wound healing in a diabetic rat burn model. J Burn Care Res 37: e115-e124, 2016.

13. García-Compeán D, Jáquez-Quintana JO, GonzálezGonzález JA, Lavalle-González FJ, Villarreal-Pérez JZ and Maldonado-Garza HJ: Diabetes in liver cirrhosis. Gastroenterol Hepatol 36: 473-482, 2013 (In Spanish).

14. Gundling F, Schepp W and Schumm-Draeger PM: Hepatogenous diabetes in cirrhosis: Academic sport or a neglected disease? Exp Clin Endocrinol Diabetes 120: 469-471, 2012.

15. Hunter SS and Hamdy S: Predictors of early re-bleeding and mortality after acute variceal haemorrhage. Arab J Gastroenterol 14: 63-67, 2013.

This work is licensed under a Creative Commons Attribution-NonCommercial-NoDerivatives 4.0 International (CC BY-NC-ND 4.0) License. 